\title{
TESTING FOR STRUCTURAL CHANGES IN EXCHANGE RATES DEPENDENCE BEYOND LINEAR CORRELATION
}

\author{
Alexandra Dias $^{a *}$ And Paul Embrechts ${ }^{b}$ \\ ${ }^{a}$ Warwick Business School, Finance Group, University of Warwick, UK \\ ${ }^{b}$ Department of Mathematics, ETH Zurich, Switzerland
}

July 16, 2007

\begin{abstract}
In this paper we test for structural changes in the conditional dependence of twodimensional foreign exchange data. We show that by modeling the conditional dependence structure using copulae we can detect changes in the dependence beyond linear correlation like changes in the tail of the joint distribution. This methodology is relevant for estimating risk management measures as portfolio Value-at-Risk, pricing multi-name financial instruments and portfolio asset allocation. Our results include evidence of the existence of changes in the correlation as well as in the fatness of the tail of the dependence between Deutsche Mark and Japanese Yen.
\end{abstract}

KEY WorDS: change-point tests; conditional dependence; copula; GARCH; risk management.

\section{Introduction}

In financial markets, the price movements of different assets are invariably related. In addition, financial crises, market deregulations, policy shifts, central bank interventions may cause changes in the way financial asset prices relate with each other. The dynamics of asset dependence is of importance in risk management, asset pricing, portfolio allocation and forecasting. Not surprisingly, there is considerable interest in the dynamic behavior of correlation between different risks as a function of time. Financial theory and models often assume that (conditional) correlation between assets are possibly time varying; see for instance the Dynamic Conditional Correlation-GARCH model from Engle (2002). Loretan and Phillips (1994) study tests for covariance stationarity, Boyer et al. (1999) investigate pitfalls in tests for changes in correlation, Longin and Solnik (2001) relate the dynamics of conditional correlation with market trend and Andreou and Ghysels (2003) analyze procedures for testing for changes in conditional correlation. There is an enormous econometric literature on the use of regime changes for describing non-stationary economic data. In the context of time series analysis; see

* Correspondence address: Alexandra Dias, Warwick Business School, Finance Group, University of Warwick, CV4 7AL Coventry, UK. E-mail: Alexandra.Dias@wbs.ac.uk 
for instance Hamilton (1990). In the field of monetary policy, regime switches may come about as a consequence of new policy implementation; see Francis and Owyang (2005). In the latter context, tools have been developed to measure the influence of both regime changes as well as policy shocks. A further series of publications concentrates on the relationship between longmemory and the existence of structural changes, so-called "spurious long memory process"; see Mikosch and Stărică (2000), Diebold and Inoue (2001), Choi and Zivot (2007), and Mikosch and Stărică (2004).

As with other financial processes, the dependence between exchange rates is exposed to changes due to general economic events. In addition, central bank interventions are likely to have a definitive impact. For the case studied here of the Deutsche Mark and the Japanese Yen prior to the introduction of the EURO, an economic event which has a strong impact on the Japanese economy, but not on the German economy can cause instability in the Yen and trigger a move of investment preferences towards the Deutsche Mark as compared with the Japanese Yen. This should weaken the dependence between the two exchange rates. On the other hand, an intervention of the Bank of Japan to ensure a depreciation of the Yen once the Deutsche Mark depreciates against the Dollar, to keep Japanese export competitive, might cause an increase in the dependence between the two exchange rates.

We present a methodology for testing for structural changes, the so-called change-points, in the conditional dependence using copulae. Through many examples, we now know that "there is more to dependence as can be measured through (linear) correlation" (Embrechts et al. (2002)). It turns out that the notion of copula yields an excellent tool for the modeling of nonlinear dependencies. These techniques have now achieved text book level; see for example Cherubini et al. (2004), Joe (1997), McNeil et al. (2005) and Nelsen (2006). Dynamic dependence structures modeled with copulae are for instance to be found in Rockinger and Jondeau (2006), Fortin and Kuzmics (2002), Patton (2006a), Patton (2006b) and Giacomini et al. (2006).

In this paper we extend existing methods in a number of ways. We assume that the multivariate asset return process is dynamic heteroskedastic and the asset returns are to be standardized by conditional volatility estimates. We take a two-stage estimation approach. We standardize each univariate return series using GARCH volatility models (see Andersen et al. (2005)), and then test for changes in the conditional dependence with parametric (copula) models. Two-stage model estimation can be found in the literature on semi-parametric modeling, as in Andreou and Ghysels (2003), or in a full-parametric modeling context as in Engle and Sheppard (2001) and Patton (2006b). The methodology we present here is new in 
two aspects. First, our procedure allows for testing for changes not only in the conditional covariance or correlation but also for changes in the complete conditional dependence. Second, we assume that both the number of changes and when they happen are unknown. The goal is to uncover structural changes which might not impact on the covariance, such as changes in the tails of the conditional distribution. A change in the conditional tail distribution is not detected when testing for changes in the correlation and has obvious consequences in any computation involving the joint distribution like tail risk measures such as Value-at-Risk or Expected Shortfall.

We analyze daily US Dollar-Deutsche Mark and US Dollar-Japanese Yen exchange rates from April 1986 till October 1998. For this period, we test for changes in the conditional dependence modeled by a parametric copula. The reason we illustrated the change point techniques introduced in this paper with a rather old exchange rate data is that it covers a period containing several interesting economic/political events which could lead to nonstationarity in the dependence and that therefore a priori one might expect change points. We also would like to stress that the methodology introduced has much wider applicability beyond this (old) foreign exchange data set. The results show clear evidence of changes in the correlation and changes in the tail dependence between the two exchange rates. One of the most relevant results is a significant change in the dependence when the Berlin wall fell on $9^{\text {th }}$ November 1989: the conditional correlation drops substantially and the tail dependence weakens. The explanation might be that such event increased the uncertainty in the German economy without having a similar impact in Japan. The burst of the Japanese asset price bubble seems to have had the opposite effect by strengthening the dependence as we find a change in that direction in the correlation in October 1990, although the tail dependence did not change. We find a further drop in the conditional correlation in June 1997 now possibly due to a bigger impact of the Asia crisis on the Japanese Yen. The last change we remark here is detected in October 1995 where the tail dependence weakens so considerably that we can statistically assume asymptotic tail independence until the end of the period covered by the data sample, October 1998.

We detect more changes in the data which are reported later in the paper but for which we did not find immediate related economic events. One possible explanation is that changes in the dependence may also occur smoothly through time instead of suddenly. We are not modeling these smooth changes. Hence, the test statistic will point to a certain date as a change-point when in reality it is a smoothly changing process. This is not necessarily a disadvantage. Assuming a constant dependence with changes at certain points will lead to 
more stable portfolio Value-at-Risk and (dynamic) asset allocation than if we use dynamic models where the dependence is allowed to change at every point in time. Modeling the conditional dependence as being constant in time, yet allowing for the possibility of breaks to occur, is a compromise between the oversimplifying assumption of constant dependence and the highly parameterized time varying dependence models. If constant models are too simple for the evidence found in data, the time varying models are statistically much more difficult to estimate. By way of an economically relevant example, in this paper we present a new tool to financial experts that we hope will have potential for a more careful, yet parsimonious analysis of financial data.

This paper proceeds as follows. In Section 2, we present the theory of change-points for copulae. In Section 3, we test for changes in the conditional dependence between the US Dollar-Deutsche Mark and US Dollar-Japanese Yen. In the same section we perform several statistical specification and goodness-of-fit tests. Section 4 contains a summary of the results and concludes the paper.

\section{Tests for structural changes in the conditional dependence}

In this section we concentrate on the theoretic methods we use for the detection of structural changes in the conditional dependence of a multivariate time series. The first step is the standardization of the one period logarithmic univariate returns, $r_{t}=p_{t}-p_{t-1}$ where $p_{t}$ stands for the logarithmic price of a financial asset at time $t \geq 1$. We decompose the return series as $r_{t}=\mu_{t}+\sigma_{t} \varepsilon_{t}$, where $\varepsilon_{t}$ are independent and identically distributed with zero mean and unit variance, and $\sigma_{t}$ is the conditional standard deviation at time $t$. As a consequence, the $\sigma$-standardized returns are given by

$$
\varepsilon_{t}=\frac{r_{t}-\mu_{t}}{\sigma_{t}}, \quad t \geq 1
$$

The process $\sigma_{t}$ is not observable and its most widely used (univariate or multivariate) estimators involve autoregressive conditional heteroskedasticity (ARCH) type models, stochastic volatility models, realized volatility measures and continuous-time models; see Andersen et al. (2005) for a systematic review of volatility measures and specific references. The test statistics reported in Section 3 confirm the existence of conditional heteroskedasticity in the data set we use. Hence, we filter the returns with ARCH-type volatility models. As these are parametric models we prevent possible erroneous inference by performing tests for model specification and structural breaks in the univariate margins in our data analysis. 
The stability of the dependence structure of the $d$-dimensional vector process of filtered returns $\varepsilon_{i t}$, for $i=1,2, \ldots, d$, is then to be tested for structural breaks. Loretan and Phillips (1994), Longin and Solnik (2001), Andreou and Ghysels (2003), among others, consider the detection of breaks in the conditional covariance. The univariate filtered returns are assumed to be independent and identically distributed (iid) and this is supported for our data by statistical tests reported in Section 3. Hence, the only possible source of instability is the dependence structure which is completely characterized by the copula as shown by Sklar (1959). We consider a parametric copula-based model for the multivariate conditional filtered returns

$$
\begin{aligned}
F\left(\varepsilon_{1 t}, \ldots, \varepsilon_{d t} ; \boldsymbol{\theta}_{t}\right) & =C\left(F_{1}\left(\varepsilon_{1 t}\right), \ldots, F_{d}\left(\varepsilon_{d t}\right) ; \boldsymbol{\theta}_{t}\right) \\
& =C\left(\mathbf{u} ; \boldsymbol{\theta}_{t}\right)
\end{aligned}
$$

where $F$ is the conditional distribution function of the filtered returns, $F_{i}(i=1,2, \ldots, d)$ are univariate continuous distribution functions of the margins, $C$ is a copula function with vector parameter $\boldsymbol{\theta}_{t}$. For ease of exposition, we concentrate on the bivariate case, $d=2$. The methodologies used here can be extended to higher dimensions by considering appropriate copula-based model.

\subsection{Detecting change-points in copula parameters}

Testing for breaks in the multivariate conditional distribution of the filtered returns is equivalent to testing for breaks in the copula. We analyze this issue through a change-point detection technique for parametric copula models. There are several well known tests on structural breaks in econometric time-series analysis; see for instance Bai (1997), Bai and Perron (1998), Hansen (2001), and Polzehl and Spokoiny (2006). Here, we test for changes in the copula parameters, estimate the size of those changes and the corresponding time of occurrence. For related work, see for instance Gombay and Horváth (1999). For a detailed treatment of the change-point theory underlying our approach, see Csörgő and Horváth (1997) and references therein, and Dias and Embrechts (2002).

Let $\mathbf{U}_{1}, \mathbf{U}_{2}, \ldots, \mathbf{U}_{n}$ be a sequence of independent random vectors in $[0,1]^{d}$ with univariate uniformly distributed margins and copulae $C\left(\mathbf{u} ; \boldsymbol{\theta}_{1}, \boldsymbol{\eta}_{1}\right), C\left(\mathbf{u} ; \boldsymbol{\theta}_{2}, \boldsymbol{\eta}_{2}\right), \ldots, C\left(\mathbf{u} ; \boldsymbol{\theta}_{n}, \boldsymbol{\eta}_{n}\right)$ respectively, where $\boldsymbol{\theta}_{i}$ and $\boldsymbol{\eta}_{i}$ are the copula parameters such that $\boldsymbol{\theta}_{i} \in \Theta^{(1)} \subseteq \mathbb{R}^{p}$ and $\boldsymbol{\eta}_{i} \in \Theta^{(2)} \subseteq \mathbb{R}^{q}$. We will consider the $\boldsymbol{\eta}_{i}$ as constant parameters and look for one single change-point in $\boldsymbol{\theta}_{i}$. Formally, we test the null hypothesis

$$
H_{0}: \boldsymbol{\theta}_{1}=\boldsymbol{\theta}_{2}=\ldots=\boldsymbol{\theta}_{n} \quad \text { and } \quad \boldsymbol{\eta}_{1}=\boldsymbol{\eta}_{2}=\ldots=\boldsymbol{\eta}_{n}
$$


versus the alternative

$$
H_{A}: \boldsymbol{\theta}_{1}=\ldots=\boldsymbol{\theta}_{k^{*}} \neq \boldsymbol{\theta}_{k^{*}+1}=\ldots=\boldsymbol{\theta}_{n} \quad \text { and } \quad \boldsymbol{\eta}_{1}=\boldsymbol{\eta}_{2}=\ldots=\boldsymbol{\eta}_{n}
$$

If we reject the null hypothesis, $k^{*}$ is the time of the change. All the parameters of the model are supposed to be unknown under both hypotheses. If $k^{*}=k$ were known, the null hypothesis would be rejected for small values of the likelihood ratio statistic

$$
\Lambda_{k}=\frac{\sup _{(\boldsymbol{\theta}, \boldsymbol{\eta}) \in \Theta^{(1)} \times \Theta^{(2)}} \prod_{1 \leq i \leq n} c\left(\mathbf{u}_{i} ; \boldsymbol{\theta}, \boldsymbol{\eta}\right)}{\sup _{\left(\boldsymbol{\theta}, \boldsymbol{\theta}^{\prime}, \boldsymbol{\eta}\right) \in \Theta^{(1)} \times \Theta^{(1)} \times \Theta^{(2)}} \prod_{1 \leq i \leq k} c\left(\mathbf{u}_{i} ; \boldsymbol{\theta}, \boldsymbol{\eta}\right) \prod_{k<i \leq n} c\left(\mathbf{u}_{i} ; \boldsymbol{\theta}^{\prime}, \boldsymbol{\eta}\right)},
$$

where we assume that $C$ has a density $c$. The estimation of $\Lambda_{k}$ is carried out through maximum likelihood. Denote

$$
L_{k}(\boldsymbol{\theta}, \boldsymbol{\eta})=\sum_{1 \leq i \leq k} \log c\left(\mathbf{u}_{i} ; \boldsymbol{\theta}, \boldsymbol{\eta}\right)
$$

and

$$
L_{k}^{*}(\boldsymbol{\theta}, \boldsymbol{\eta})=\sum_{k<i \leq n} \log c\left(\mathbf{u}_{i} ; \boldsymbol{\theta}, \boldsymbol{\eta}\right) .
$$

Then the likelihood ratio equation can be written as

$$
-2 \log \left(\Lambda_{k}\right)=2\left(L_{k}\left(\hat{\boldsymbol{\theta}}_{k}, \hat{\boldsymbol{\eta}}_{k}\right)+L_{k}^{*}\left(\boldsymbol{\theta}_{k}^{*}, \hat{\boldsymbol{\eta}}_{k}\right)-L_{n}\left(\hat{\boldsymbol{\theta}}_{n}, \hat{\boldsymbol{\eta}}_{n}\right)\right) .
$$

As $k$ is unknown, $H_{0}$ will be rejected for large values of

$$
Z_{n}=\max _{1 \leq k<n}\left(-2 \log \left(\Lambda_{k}\right)\right)
$$

\subsubsection{Asymptotic critical values}

The asymptotic distribution of $Z_{n}^{1 / 2}$ is known but has a very slow rate of convergence; see Csörgö and Horváth (1997), page 22. In the same reference we can also find an approximation for the distribution of $Z_{n}^{1 / 2}$ derived to give better small sample rejection regions. Indeed, for $0<h(n) \leq l(n)<1$, the following approximation holds

$$
\begin{aligned}
& P\left(Z_{n}^{1 / 2} \geq x\right) \approx \frac{x^{p} \exp \left(-x^{2} / 2\right)}{2^{p / 2} \Gamma(p / 2)} . \\
& \quad\left(\log \frac{(1-h)(1-l)}{h l}-\frac{p}{x^{2}} \log \frac{(1-h)(1-l)}{h l}+\frac{4}{x^{2}}+O\left(\frac{1}{x^{4}}\right)\right),
\end{aligned}
$$

as $x \rightarrow \infty$ and where $h$ and $l$ can be taken as $h(n)=l(n)=(\log n)^{3 / 2} / n$. Note that in (3) $p$ is the number of parameters that may change under the alternative. This result turns out to be very accurate as shown in a simulation study in Dias and Embrechts (2002) where it is applied to the Gumbel copula. 


\subsubsection{The time of the change}

If we assume that there is exactly one change-point, then the maximum likelihood estimator for the time of the change is given by

$$
\hat{k}_{n}=\min \left\{1 \leq k<n: Z_{n}=-2 \log \left(\Lambda_{k}\right)\right\}
$$

In the case that there is no change, $\hat{k}_{n}$ will take a value near the boundaries of the sample. This holds because under the null hypothesis, and given that all the necessary regularity conditions hold, for $n \rightarrow \infty, \hat{k}_{n} / n \stackrel{d}{\longrightarrow} \xi$, where $P(\xi=0)=P(\xi=1)=1 / 2$; see Csörgő and Horváth (1997), page 51. This behavior was verified in a simulation study for the Gumbel copula under the no-change hypothesis in Dias and Embrechts (2002).

\subsubsection{Multiple Changes}

In the case that more than one change-point exists, we use a sequential procedure coupled with hypothesis testing proposed by Vostrikova (1981) in the context of multidimensional processes with unknown parameters. This method is studied for detecting multiple breaks (one at a time) in the mean of a process in Bai (1997). It is introduced as a computationally advantageous alternative to the simultaneous estimation in Bai and Perron (1998) and detects simultaneously the number and the location of the change-points. The sequential method consists of first applying the likelihood ratio test for one change. If $H_{0}$ is rejected then we have the estimate of the time of the change $\hat{k}_{n}$. Next, we divide the sample in two subsamples $\left\{\mathbf{u}_{t}: 1 \leq t \leq \hat{k}_{n}\right\}$ and $\left\{\mathbf{u}_{t}: \hat{k}_{n}<t \leq n\right\}$ and test $H_{0}$ for each one of them. If we find a change-point in any of the sets we continue this segmentation procedure until we do not reject $H_{0}$ in any of the subsamples. Given the existence of multiple change-points and different change-sizes, the method might over- or underestimate the location of a change-point; see Bai (1997). To overcome this question Bai (1997) proposes a fine-tuning repartition procedure after estimating the $m$ change-points $\hat{k}_{1}<\hat{k}_{2}<\ldots<\hat{k}_{m}$ which we use here. Accordingly, each change-point $\hat{k}_{i}$ is reestimated by applying the test to the subsample $\left\{\mathbf{u}_{t}: \hat{k}_{i-1}+1 \leq t \leq \hat{k}_{i+1}\right\}$, where $\hat{k}_{0}=0$ and $\hat{k}_{m+1}=n$. 


\section{The conditional dependence of US Dollar-Deutsche Mark and US Dollar-Japanese Yen}

We analyze here the bivariate daily logarithmic returns ${ }^{1}$ of US Dollar-Deutsche Mark (USDDEM) and US Dollar-Japanese Yen (USD-JPY) exchange rates. The observations cover the period from $27^{\text {th }}$ April 1986 until $25^{\text {th }}$ October 1998. The data set was kindly provided by Wolfgang Breymann and Olsen Data and previously treated and cleaned as explained in Breymann et al. (2003). After the data cleaning the returns data set consists of 3259 daily observations. Table 1 contains some summary statistics. From these statistics we observe that both series show a nonsignificant trend, have negative skewness and excess kurtosis ${ }^{2}$. The unconditional linear correlation estimate yields a significant correlation between the two series. In order to test for conditional heteroscedasticity, we consider the $\mathrm{ARCH}$ effects test proposed by Engle (1982). For the two series the hypothesis of no ARCH effects has to be rejected. Our goal in this section is to model the conditional dependence underlying the bivariate

Table 1: Summary statistics

\begin{tabular}{lcc}
\hline \hline & USD-DEM & USD-JPY \\
\hline Mean & -0.0112 & -0.0128 \\
Standard deviation & 1.0182 & 1.0026 \\
Skewness & -0.1234 & -0.3171 \\
Kurtosis & 4.6838 & 5.7735 \\
Linear correlation & \multicolumn{2}{c}{0.6243} \\
\multicolumn{2}{c}{ No ARCH effects: LM test } \\
\hline USD-DEM & USD-JPY \\
\hline Pest statistic & 35.25 & 75.99 \\
P-value & 0.000 & 0.000 \\
\hline
\end{tabular}

Summary statistics for the USD-DEM and USD-JPY returns and test of the null hypothesis of no ARCH effects from Engle (1982).

USD-DEM, USD-JPY exchange rate returns. By now, a standard approach is based on the notion of conditional copula, as discussed in Patton (2006b). In this two-stage procedure, one first models the marginal dynamics and then the dependence structure.

\footnotetext{
${ }^{1}$ In the remaining of the paper we refer to the daily logarithmic returns simply as the returns.

${ }^{2}$ The values for the kurtosis given are to be compared with a value of 3 for the standard normal distribution.
} 


\subsection{Modeling the univariate margins}

The marginal tests presented in Table 1 reveal the presence of time-varying variance and heavy tailedness. In our discrete-time setting, we model stochastic volatility effects by GARCH type models. In particular, we fit univariate ARMA-GARCH models to each marginal series.

Formally, consider the sequence of iid random variables with zero mean and unit variance $\left(\varepsilon_{t}\right)_{t \in \mathbb{Z}}$. The process $\left(r_{t}\right)_{t \in \mathbb{Z}}$ is an $\operatorname{ARMA}\left(p_{1}, q_{1}\right)-\operatorname{GARCH}\left(p_{2}, q_{2}\right)$ if it satisfies the equations

$$
\begin{aligned}
r_{t} & =\mu_{t}+\epsilon_{t} \\
\mu_{t} & =\mu+\sum_{i=1}^{p_{1}} \phi_{i}\left(r_{t-i}-\mu\right)+\sum_{j=1}^{q_{1}} \theta_{j} \epsilon_{t-j} \\
\epsilon_{t} & =\sigma_{t} \varepsilon_{t} \\
\sigma_{t}^{2} & =\alpha_{0}+\sum_{i=1}^{p_{2}} \alpha_{i}\left(\left|\epsilon_{t-i}\right|+\gamma_{i} \epsilon_{t-i}\right)^{2}+\sum_{j=1}^{q_{2}} \beta_{j} \sigma_{t-j}^{2}
\end{aligned}
$$

where $\alpha_{0}>0, \alpha_{i} \geq 0$ for $i=1,2, \ldots, p_{2}, \beta_{j} \geq 0$ for $j=1,2, \ldots, q_{2}$ and $\varepsilon_{t}$ is independent

of $\left(r_{s}\right)_{s \leq t}$. The parameter $\gamma$ takes into account that innovations of different signs may have asymmetric impacts on the future variance; see for example Bollerslev et al. (1992) and references therein. We fit univariate ARMA-GARCH models by maximum likelihood to each marginal series assuming that the innovations $\varepsilon_{t}$ come from a Student- $t$ distribution with $\nu$ degrees of freedom. Table 2 presents the estimates of the parameters of the models with the respective asymptotic standard errors in parenthesis. The moving average component could be removed for either series. The USD-DEM data require an autoregressive lag of order one and the USD-JPY needs an autoregressive lag of order ten. The necessary lags for the ARCH and GARCH components are both of order one for the two exchange rates. For the USD-DEM returns we cannot reject the null hypothesis of $\gamma_{i}=0$ for the estimated asymmetry parameter and in the case of USD-JPY, we reject the same null hypothesis. The rejection of $\gamma_{i}=0$ for the USD-JPY, given that the estimate value $\hat{\gamma}$ is negative, indicates that negative shocks on the USD-JPY rate have a larger impact on future volatility than positive shocks.

From the fitted ARMA-GARCH model parameters we recover the residuals or filtered returns $\hat{\varepsilon}_{t}$ for each univariate time series $\left(r_{1}, r_{2}, \ldots, r_{n}\right)$ :

$$
\hat{\varepsilon}_{t}=\left(r_{t}-\hat{\mu}_{t}\right) / \hat{\sigma}_{t}, \quad t=1,2, \ldots, n
$$

Once the univariate models are selected and fitted, the dynamics as well as the goodnessof-fit of the t-density, must be checked. We use the Ljung-Box (L-B) and the the multivariate Portmanteau test, from Hosking (1980), for testing for serial correlation on the filtered returns 
Table 2: Univariate modeling of the USD-DEM and USD-JPY returns

\begin{tabular}{lcc}
\hline \hline & $\begin{array}{c}\text { USD-DEM } \\
\text { estimate(s.e. })\end{array}$ & $\begin{array}{c}\text { USD-JPY } \\
\text { estimate(s.e. })\end{array}$ \\
\hline Constant, $\hat{\mu}$ & $-0.0046(0.0161)$ & $0.0226(0.0153)$ \\
AR $(1), \hat{\phi}_{1}$ & $-0.0343(0.0165)$ & - \\
AR $(10), \hat{\phi}_{10}$ & - & $0.0597(0.0163)$ \\
GARCH constant, $\hat{\alpha}_{0}$ & $0.0423(0.0185)$ & $0.1267(0.0481)$ \\
Lagged $\epsilon^{2}, \hat{\alpha}_{1}$ & $0.0348(0.0088)$ & $0.0726(0.0213)$ \\
Lagged variance, $\hat{\beta}_{1}$ & $0.9160(0.0215)$ & $0.7927(0.0623)$ \\
Asymmetry, $\hat{\gamma}_{1}$ & - & $-0.1557(0.0801)$ \\
Degrees of freedom, $\hat{\nu}$ & $5.7967(0.5557)$ & $4.8894(0.4119)$ \\
\hline
\end{tabular}

This table shows the maximum likelihood estimates and corresponding asymptotic standard errors obtained from fitting ARMA-GARCH models to each of the return series USD-DEM and USD-JPY.

up to the fourth moment and on the absolute values of the residuals. With the AndersonDarling (A-D) test we assess the goodness-of-fit of the t-density and with Jarque-Bera test the normality of the filtered returns. We also test for heteroscedasticity or ARCH effects and for the existence of structural breaks on the univariate filtered returns. In Table 3 we report the p-values obtained for these tests.

Table 3: Specification tests on the filtered returns (p-values)

\begin{tabular}{lccc}
\hline \hline Null hypothesis & USD-DEM & USD-JPY & Multivariate test \\
\hline No serial correlation of 1st moment & 0.1204 & 0.5328 & 0.0614 \\
No serial correl of absol values & 0.8456 & 0.6803 & 0.8525 \\
No serial correlation of 2nd moment & 0.9702 & 0.9154 & 0.9690 \\
No serial correlation of 3rd moment & 0.1225 & 0.2474 & 0.5524 \\
No serial correlation of 4th moment & 0.3969 & 0.2942 & 0.9481 \\
No ARCH effects & 0.9663 & 0.9183 & - \\
Normally distributed & 0.0000 & 0.0000 & - \\
Student- $t$ distributed & 0.6452 & 0.3105 & - \\
No structural breaks & 0.5829 & 0.1025 & - \\
\hline
\end{tabular}

Here are shown the p-values obtained when testing for serial correlation, cross correlation, ARCH effects and structural breaks in the filtered returns of USD-DEM and USD-JPY. The p-values of the normality Jarque-Bera and Student- $t$ Anderson-Darling goodness-of-fit tests are also given.

The $\mathrm{L}-\mathrm{B}$ and the Portmanteau tests give indication of no serial or cross correlation. There is no evidence of remaining ARCH effects. The goodness-of-fit tests for the marginal distribu- 
tions reject normality, according to the $\mathrm{J}-\mathrm{B}$ test, and do not reject the Student- $t$ distribution, according to the A-D test. That confirms our choice of a Student- $t$ for the innovations of the models. We also tried to include crossed lagged returns in these models but without success. We also tested the existence of change-points in the parameters of the distribution of the univariate filtered returns. If the marginal models are appropriate then the filtered returns should come from a Student- $t$ distribution with constant parameters (location, scale and degrees of freedom). Given the results for non serial correlation reported in Table 3 we assume each univariate filtered returns series to be independent. We test the null hypothesis of no changepoints in any of the three parameters of the univariate Student- $t$ applying (2) and (3). For the USD-DEM we obtain a test statistic value of $z_{n o b s}^{1 / 2}=3.195$ to which corresponds a p-value of 0.5829 according to (3). In the case of the USD-JPY filtered returns we have that $z_{\text {nobs }}^{1 / 2}=3.880$ which implies a p-value of 0.1025 . In both cases we do not reject the no change-points hypothesis. On the other hand, there is a significant contemporaneous linear correlation between the two filtered time series. The estimated linear correlation is $\hat{\rho}=0.6180$ which turns out to be of the same order as the one obtained for the (non-filtered) returns. It is this dependence that we want to model using copulae. In the next section we perform a copula analysis of the bivariate filtered returns.

\subsection{Modeling the conditional dependence structure: copula}

In this section we model the dependence structure or copula between the two exchange rates USD-DEM and USD-JPY filtered returns. There are many parametric families of copulae; see Nelsen (2006), Joe (1997) and McNeil et al. (2005). As we do not know at this stage whether there are or not changes in the conditional dependence, we first perform a static copula analysis in order to restrict the class of parametric copula families we want to concentrate on. Later in the analysis, once the change-points are detected we check the appropriateness of the copula model again (see Section 3.4).

Assuming at first stationarity, suppose that the USD-DEM filtered returns are represented by $\varepsilon_{1}$ and the USD-JPY by $\varepsilon_{2}$. Assume that $\left(\varepsilon_{1}, \varepsilon_{2}\right)$ has multivariate distribution function $F$ and continuous univariate marginal distribution functions $F_{1}$ and $F_{2}$. In order to investigate the dependence, we fit copula-based models of the type

$$
F\left(\varepsilon_{1}, \varepsilon_{2} ; \boldsymbol{\theta}\right)=C\left(F_{1}\left(\varepsilon_{1}\right), F_{2}\left(\varepsilon_{2}\right) ; \boldsymbol{\theta}\right),
$$

where $C$ is a copula function, which we know to exist uniquely by Sklar's Theorem (Sklar (1959)), parameterized by the vector $\boldsymbol{\theta} \in \mathbb{R}^{q}$ with $q \in \mathbb{N}$. The corresponding model density is 
the product of the copula density $c$ and the marginal densities $f_{1}$ and $f_{2}$ :

$$
f\left(\varepsilon_{1}, \varepsilon_{2} ; \boldsymbol{\theta}\right)=c\left(F_{1}\left(\varepsilon_{1}\right), F_{2}\left(\varepsilon_{2}\right) ; \boldsymbol{\theta}\right) f_{1}\left(\varepsilon_{1}\right) f_{2}\left(\varepsilon_{2}\right),
$$

where $c$ is the copula density of model (7) and is given by

$$
c\left(u_{1}, u_{2} ; \boldsymbol{\theta}\right)=\frac{\partial^{2} C\left(u_{1}, u_{2} ; \boldsymbol{\theta}\right)}{\partial u_{1} \partial u_{2}}, \quad\left(u_{1}, u_{2}\right) \in[0,1]^{2} .
$$

Denote by $\left\{\left(\varepsilon_{1 i}, \varepsilon_{2 i}\right): i=1,2, \ldots, n\right\}$ a general random sample of $n$ bivariate observations. The marginal distribution functions $F_{i}, i=1,2$, are estimated by the rescaled empirical distribution functions $F_{i n}(z)=\frac{1}{n+1} \sum_{j=1}^{n} \mathbb{I}_{\{y \in \mathbb{R}: y \leq z\}}\left(\varepsilon_{i j}\right)$. As usual $\mathbb{I}_{A}$ denotes the indicator function of the set $A$. After the marginal transformations to the so-called pseudo observations $\left(F_{1 n}\left(\varepsilon_{1 i}\right), F_{2 n}\left(\varepsilon_{2 i}\right)\right)$ for $i=1,2, \ldots, n$, the copula family $C$ is fitted. Suppose that its density exists, we then maximize the pseudo log-likelihood function

$$
L(\boldsymbol{\theta} ; \boldsymbol{\varepsilon})=\sum_{i=1}^{n} \log c\left(F_{1 n}\left(\varepsilon_{1 i}\right), F_{2 n}\left(\varepsilon_{2 i}\right) ; \boldsymbol{\theta}\right) .
$$

The dependence parameter $\hat{\boldsymbol{\theta}}$ which maximizes (8) is the pseudo log-likelihood estimate introduced by Genest et al. (1995) in the iid case and further studied by Chen and Fan (2005) in the non-iid case. Note however that the preliminary ARMA-GARCH filtering may increase the variance of the estimates of $\boldsymbol{\theta}$.

The copula families considered are: t, Frank, Plackett, Gaussian, Gumbel, Clayton, symmetrized Joe-Clayton and the mixtures Gumbel with survival Gumbel, Clayton with survival Clayton, Gumbel with Clayton and survival Gumbel with survival Clayton; for details on these classes see Joe (1997), Nelsen (2006), Embrechts et al. (2002), McNeil et al. (2005) and Patton (2006b). Denoting the copula family $A$ with parameter $\boldsymbol{\theta}$ by $C^{A}(\cdot, \cdot ; \boldsymbol{\theta})$, the fitted mixtures have distribution functions of the form

$$
C\left(u_{1}, u_{2} ; \boldsymbol{\theta}\right)=\theta_{3} C^{A}\left(u_{1}, u_{2} ; \theta_{1}\right)+\left(1-\theta_{3}\right) C^{B}\left(u_{1}, u_{2} ; \theta_{2}\right) .
$$

The above choice of copula models is partly based on previous analyses, on tractability and flexibility, on methodological results and also to allow for a fairly broad class with respect to extremal clustering and possible asymmetry. The Gaussian copula is included mainly for comparison. The models were ranked using the Akaike information criterion

$$
\mathrm{AIC}=-2 L(\hat{\boldsymbol{\theta}} ; \boldsymbol{\varepsilon})+2 k
$$

and the Bayesian information criterion

$$
\mathrm{BIC}=-2 \ln (L(\hat{\boldsymbol{\theta}} ; \boldsymbol{\varepsilon}))+k \ln (n)
$$


where $n$ is the number of observations and $k$ is the number of parameters of the family fitted. Parameter estimates and asymptotic standard errors (s.e.) for all fitted models are listed in Table 4. For the four mixture models the parameters $\theta_{1}, \theta_{2}$ and $\theta_{3}$ are as in (9). For the symmetrized Joe-Clayton copula, $\theta_{1}$ and $\theta_{2}$ are the lower and upper tail parameters respectively. For the $t$-copula, $\theta_{1}$ represents the degrees of freedom and $\theta_{2}$ represents the correlation. The mixture of Gumbel and survival Gumbel and the $t$-copula are the best ranked models. While the Gumbel mixture model allows for asymmetry, the $t$-copula is symmetric. We test for asymmetry of the Gumbel mixture. As the AIC of these two models is close and the BIC favours the $t$-copula, if the Gumbel mixture reveals to be symmetric then we prefer to use the $t$-model because of the economic interpretation of its parameters and because it is very well known in the econometric literature. We perform a likelihood ratio (LR) test for possible asymmetry in the Gumbel mixture model (9); we tested for the null hypothesis

$$
H_{0}: \theta_{1}=\theta_{2} \quad \text { and } \quad \theta_{3}=0.5
$$

versus the alternative

$$
H_{A}: \theta_{1} \neq \theta_{2} \quad \text { or } \quad \theta_{3} \neq 0.5
$$

where $\theta_{1}, \theta_{2}$ and $\theta_{3}$ are the scalar parameters of the model. A low p-value indicates that a three parameter asymmetric Gumbel mixture model is significantly better than the one parameter symmetric model. This turns out not to be the case. We obtain a p-value of 0.1842 favoring the symmetric model. This may seem in contrast to Patton (2006b) where for DEM-USD and JPY-USD daily data, the symmetrized Joe-Clayton copula model indicates asymmetry. We also test for asymmetry in the symmetrized Joe-Clayton copula model for our data set. We obtain a p-value of 0.641 rejecting the hypothesis of asymmetry. A comparison of these results may not be straightforward because the two data sets cover different periods. Based on the above we continue the analysis of our data with a $t$-copula model. Further support for the $t$-based models is to be found in Breymann et al. (2003), Demarta and McNeil (2005), McNeil et al. (2005), Daul et al. (2003), Rosenberg and Schuermann (2006) and Pesaran et al. (2004).

\subsection{Testing for structural changes in the conditional dependence between DEM and JPY}

In this section we test for the occurrence of change-points in the dependence structure of the conditional distribution of USD-DEM and USD-JPY modeled by a $t$-copula. Specifically, we test for change-points in the copula parameters of the filtered returns of USD-DEM and USD-JPY exchange rates data. Concretely we use the procedures from Section 2 to estimate 
Table 4: Conditional dependence: copula modeling

\begin{tabular}{lccccc}
\hline \hline & $\hat{\theta}_{1}$ (s.e. $)$ & $\hat{\theta}_{2}$ (s.e. $)$ & $\hat{\theta}_{3}$ (s.e.) & AIC & BIC \\
\hline Clayton & $1.034(0.035)$ & - & - & -1252.289 & -1246.200 \\
Frank & $4.599(0.124)$ & - & - & -1446.464 & -1440.375 \\
Gumbel & $1.679(0.023)$ & - & - & -1500.065 & -1493.976 \\
Plackett & $7.772(0.350)$ & - & - & -1526.993 & -1520.904 \\
Gaussian & $0.617(0.009)$ & - & - & -1552.695 & -1546.606 \\
Clayton \& survival Clayton & $1.548(0.120)$ & $1.280(0.099)$ & $0.494(0.032)$ & -1599.798 & -1581.530 \\
Symmetrized Joe-Clayton & $0.417(0.018)$ & $0.430(0.017)$ & - & -1603.307 & -1591.129 \\
Clayton \& Gumbel & $1.665(0.045)$ & $1.844(0.249)$ & $0.671(0.037)$ & -1629.394 & -1611.126 \\
Surv. Clayton \& surv. Gumbel & $1.816(0.071)$ & $1.234(0.195)$ & $0.656(0.039)$ & -1632.435 & -1614.167 \\
Gumbel \& survival Gumbel & $1.588(0.072)$ & $1.952(0.117)$ & $0.501(0.048)$ & -1642.460 & -1624.192 \\
$t$-copula & $6.012(0.786)$ & $0.620(0.010)$ & - & -1640.061 & -1627.883 \\
\hline
\end{tabular}

Results from fitting copula models to the filtered returns of USD-DEM and USD-JPY. The table shows the model parameter estimates and the asymptotic standard errors. In case of the mixture copulae, $\theta_{1}$ and $\theta_{2}$ are the dependence parameters respectively for the first and second terms of the mixture. $\theta_{3}$ is the mixture parameter which gives the proportion of the first term. For the $t$-copula, $\theta_{1}$ stands for the degrees of freedom and $\theta_{2}$ is the correlation. The last two columns display the information criteria AIC and BIC obtained for each model.

change-points in the correlation and degrees of freedom parameters of a $t$-copula fitted to the filtered returns. For the change-points found, we estimate the size of those changes and the corresponding time of occurrence. We also look for economic events that may have triggered these changes. After filtering the univariate returns using the GARCH type models reported in Table 2 of Section 3.1, given the results in Table 3 we can assume the filtered returns to be a sequence of independent bivariate vectors with no breaks in the univariate margins. Hence, we satisfy the conditions for testing for the existence of change-points in the copula parameters. We use the methods specified in Section 2.1 for detecting possible change-points in the parameters of the multivariate contemporaneous conditional distribution.

In the first step, we test for change-points assuming that one or two parameters may change: degrees of freedom and/or correlation. This corresponds to $p=2$ in expression (3). We evaluate $\Lambda_{k}$ for $k=1,2, \ldots, n$ where $n=3259$; see (1). The test statistic (2) takes the value $z_{n \text { obs }}^{1 / 2}=13.49$ and by $(3)$ we have that $P\left(Z_{n}^{1 / 2}>13.49\right) \approx 0$. The null hypothesis of no change-point is to be rejected and the estimated time of the change is $\hat{k}_{n}=8^{\text {th }}$ November 1989; coinciding with the fall of the Berlin wall. See Table 5 row corresponding to $\mathbf{I}$ in the 
first column. The values obtained in this and subsequent tests are displayed in Table 5. Next, the sample is divided in two sub-samples, one up to $8^{\text {th }}$ November 1989 and another from the estimated time of change onwards. For each sub-sample $\Lambda_{k}$ is computed as well as $Z_{n}^{1 / 2}$. The rows corresponding to II in the first column of Table 5 have those values. As the obtained p-values are close to zero we reject the null hypothesis of no change for each sub-sample and estimate two more change-points, $29^{\text {th }}$ December 1986 and $9^{\text {th }}$ June 1997 . The later date corresponds to the beginning of the Asia crisis starting with the devaluation of the Thai Baht. Each sub-sample is again divided in two and the procedure is repeated yielding the estimates in the rows corresponding to III. For these results, only for the maximum attained at $23^{\text {rd }}$

Table 5: Tests for change-points in the conditional dependence

\begin{tabular}{crrccc}
\hline \hline & $z_{n \text { obs }}^{1 / 2}$ & \multicolumn{1}{c}{$n$} & $P\left(Z_{n}^{1 / 2}>z_{n \text { obs }}^{1 / 2}\right)$ & $H_{0}(0.95)$ & Time of change \\
\hline \hline I & 13.49 & 3259 & 0.0000000 & reject & 8 Nov. 1989 \\
\hline II & 6.21 & 922 & 0.0000006 & reject & 29 Dec. 1986 \\
& 5.51 & 2337 & 0.0000330 & reject & 9 June 1997 \\
\hline III & 3.28 & 176 & 0.1133105 & not rej. & - \\
& 3.02 & 746 & 0.3006019 & not rej. & - \\
& 5.87 & 1979 & 0.0000047 & reject & 23 Oct. 1990 \\
& 2.35 & 358 & 0.9999999 & not rej. & - \\
\hline IV & 2.71 & 249 & 0.4409689 & not rej. & - \\
& 4.25 & 1730 & 0.0087136 & reject & 18 Oct. 1995 \\
\hline V & 5.99 & 1302 & 0.0000022 & reject & 26 Sept. 1994 \\
& 2.87 & 428 & 0.3620263 & not rej. & - \\
\hline VI & 3.06 & 1023 & 0.2917965 & not rej. & - \\
& 2.21 & 279 & 0.9216038 & not rej. & - \\
\hline
\end{tabular}

Change-point analysis for USD-DEM and USD-JPY conditional dependence. In the column "Time of change" the dates are the change-points estimated in at least one of the dependence parameters.

October 1990 the null hypothesis is rejected at a 5\% level. This change-point might be related with the burst in the Japanese asset price bubble. Next we have to split the sub-sample $8^{\text {th }}$ November 1989 till $9^{\text {th }}$ June 1997 in two at the date $23^{\text {rd }}$ October 1990 . From these two tests we estimate a change-point in $18^{\text {th }}$ October 1995 . So we still have to split this sub-sample further. The first from $23^{\text {rd }}$ October 1990 until $18^{\text {th }}$ October 1995 and the second from this date up to $9^{\text {th }}$ June 1997. The last change-point is estimated at $26^{\text {th }}$ September 1994 . Testing in further sub-samples does not lead to a rejection of the no change-point hypothesis. These values are in the rows corresponding to VI of Table 5. In summary we found six change- 
points: $29^{\text {th }}$ December $1986,8^{\text {th }}$ November $1989,23^{\text {rd }}$ October $1990,26^{\text {th }}$ September 1994 , $18^{\text {th }}$ October 1995 and $9^{\text {th }}$ June 1997.

Three questions have to be raised now. The detected change-points were obtained in a sequence of tests where the boundaries of the sub-samples depend on the change-points detected in the previous tests, except for the first test performed on the full sample. Also multiple change-points and different change-sizes may induce the tests to under- or overestimate the location of the change-point. We solve this question by applying the fine-tuning repartition procedure from Bai (1997) as explained in Section 2.1.3. Hence, each change-point is reestimated by testing on the sub-sample which has only that particular change-point according to the first sequence of tests. The second question has to do with the sub-sample sizes. As the boundaries of the sub-samples are defined by the estimated change-points each sub-sample has a different size. That is not a problem because the approximation for the distribution of the test statistic (3) takes the sample size into account. Yet, some sub-samples might have a small size which raises the question of the power of the test. The smallest sub-sample tested has size $n=176$ and we obtained good results for the power of the test in case of smaller samples with $n=100$ in our previous work Dias and Embrechts (2002). For this reason, although this question would deserve a full study by itself, we are confident about the results obtained here. The third question is a reminder that in the sequence of tests performed the null hypothesis considers that the two parameters may change. The implication is that the fine-tuning repartition procedure has to be applied twice. Once, where we test for a change-point in the correlation and constant degrees of freedom. Second, where we test for a change-point in the degrees of freedom and constant correlation. Using this procedure, besides checking the location of the change-point we identify if the change occurred in the correlation, in the degrees of freedom or in both parameters of the $t$-copula model.

Table 6 reports the results from the fine-tuning procedure. The change-point estimated in $29^{\text {th }}$ December 1986 is identified to have occurred in the correlation only. On the other hand the second estimated change-point turns out to be a change-point in the correlation and in the degrees of freedom. This change seems to be related with the fall of the Berlin wall in $9^{\text {th }}$ November 1990. The $23^{\text {rd }}$ October 1990 is found to have been a change-point in the correlation. It is interesting to note that the former date $\left(23^{\text {rd }}\right.$ October 1990) corresponds to the burst in the Japanese asset price bubble. On $18^{\text {th }}$ October 1990, the USD-JPY ended a fall from about 158 to 125. The change-points estimated in September/October 1994 and October 1995 are change-points in the degrees of freedom and in the correlation. Finally, in July 1997 we estimate a change-point in the correlation only. This date corresponds to the 
Table 6: Tests for change-points in the conditional dependence parameters

\begin{tabular}{cccc}
\hline \hline \multicolumn{4}{c}{ Breaks in the t-copula correlation, $\rho$} \\
\hline$z_{n \text { obs }}^{1 / 2}$ & $n$ & $P\left(Z_{n}^{1 / 2}>z_{n \text { obs }}^{1 / 2}\right)$ & Time of change \\
\hline 3.70 & 922 & 0.0000003 & 29 Dec. 1986 \\
11.93 & 997 & 0.0000000 & 8 Nov. 1989 \\
5.01 & 1273 & 0.0000573 & 23 Oct. 1990 \\
5.85 & 1302 & 0.0000006 & 26 Sept. 1994 \\
6.51 & 707 & 0.0000000 & 24 Oct 1995 \\
3.94 & 787 & 0.0047889 & 1 July 1997 \\
\hline & & & \\
\hline$z_{n \text { obs }}^{1 / 2}$ & $n$ & $P\left(Z_{n}^{1 / 2}>z_{n \text { obs }}^{1 / 2}\right)$ & Time of change \\
\hline 3.72 & 997 & 0.0111743 & 30 Oct. 1989 \\
3.83 & 1302 & 0.0078626 & 17 Oct. 1994 \\
4.50 & 707 & 0.0005187 & 16 Oct. 1995
\end{tabular}

Results from the fine-tuning procedure reestimating the change-points on the conditional dependence between USD-DEM and USD-JPY. At the same time we test for each change-point which of the two $t$-copula model parameters changed. In the top panel are displayed the change-points in the correlation and in the bottom panel are the change-points in the degrees of freedom. Remark the closeness of the change-point in the correlation in $8^{\text {th }}$ November 1989 and the change-point in the tail parameter in $30^{\text {th }}$ October 1989. These two breaks might be related with the fall of the Berlin wall in $9^{\text {th }}$ October 1989.

beginning of the Asia crisis starting with the devaluation of the Thai Baht. We did not find particular economic events possibly associated with some of the change-points detected. A reason for this could be that smooth rather than sudden changes can occur in the dependence structure. In these cases we do not expect to find an event justifying a sudden change in the dependence. Hence, in case of smooth changes we are modeling the dependence dynamics with jumps at a certain points in time but otherwise constant. This approach can have advantages in terms of its applications when compared with a time-varying modeling where changes occur at every point in time. This is the case, for instance, of the DCC-GARCH or BEKK model from Engle and Kroner (1995). The change-point approach will give more stable portfolio Value-at-Risk estimates with changes at a certain points when compared with a time-varying correlation model. Dynamic portfolio asset allocation and pricing are other examples where the same reasoning applies.

The test for change-points, besides detecting the time of the change, also allows to compute 
the size of the change. Let us consider now that aspect of our empirical study. For the periods between the times of changes we estimated the parameters of the $t$-copula model. Although the change-points estimated for the degrees of freedom are close to change-points estimated for the correlation, they do not coincide precisely. For this reason we exclude the small periods between those from the fitting procedure. Specifically we do not consider the observations in the periods: $10^{\text {th }}$ October 1989 till $11^{\text {th }}$ November 1989; 26 $6^{\text {th }}$ September 1994 till $17^{\text {th }}$ October 1994; and $16^{\text {th }}$ October 1995 till $24^{\text {th }}$ October 1995. Table 7 has the estimates for the correlation and degrees of freedom obtained for each sub-sample. We look for an interpretation for the raises and falls estimated in the correlation and in the degrees of freedom (or fatness of the tail distribution). A change in the dependence structure must be the result of an event, economic variable or policy change which impacts more in the Deutsche Mark than in the Japanese Yen or vice versa. For instance, if the Deutsche Mark falls against the US Dollar the Japanese central bank might impose a fall in the Yen in order to keep the competitiveness of its exportations. This would induce a strengthening of the dependence between the two exchange rates. A raise in the Yen against Deutsche Mark might be stopped also by the central bank with the same consequences for the dependence. The change in the correlation from 0.315 to 0.585 estimated in October, 1990 around the burst of the Japanese asset prices seems to be an example. On the other hand, an event or developments which might anticipate instability in the German economy say, but not in the Japanese economy should originate a weakening in the dependence between Mark and Yen. In fact, by the time of the fall of the Berlin wall we estimate a reduction in the correlation from 0.832 to 0.315 . The Asia crisis seems to have had a similar effect as the correlation decreased from 0.556 to 0.348 in July of 1997 .

As a function of the correlation and degrees of freedom, the coefficient of asymptotic tail dependence for the $t$-copula model (see Embrechts et al. (2002)) takes the form

$$
\lambda(\rho, \nu)=2 \bar{t}_{\nu+1}\left(\sqrt{\nu+1} \frac{\sqrt{1-\rho}}{\sqrt{1+\rho}}\right),
$$

where $\bar{t}_{\nu+1}$ denotes the tail, or survival function, of a univariate Student- $t$ distribution with $\nu+1$ degrees of freedom. The estimated values for the coefficient of asymptotic tail dependence are in the last column of Table 7. We can observe that there is a real impact on the tail dependence when there are changes in the correlation and degrees of freedom. The most notable impact is when the degrees of freedom estimate changes to infinity in October 1995. From there onwards we can assume asymptotic tail independence between the two exchange rates. In terms of Value-at-Risk this means a reduction in the quantile from a heavy tailed to a thin tailed distribution. 
Table 7: Conditional dependence parameters and coefficient of tail dependence $\lambda$, estimated between change-points

\begin{tabular}{|c|c|c|}
\hline \multicolumn{3}{|c|}{$t$-copula correlation $\rho$, and coefficient of tail dependence $\lambda$} \\
\hline Period & $\hat{\rho}$ (s.e.) & $\lambda(\hat{\rho}, \hat{\nu})$ \\
\hline 27 April 1986 till 29 Dec. 1986 & $0.634(0.042)$ & 0.288 \\
\hline 29 Dec. 1986 till 30 Oct. 1989 & $0.832(0.010)$ & 0.482 \\
\hline 8 Nov. 1989 till 23 Oct. 1990 & $0.315(0.059)$ & 0.053 \\
\hline 23 Oct. 1990 till 26 Sept. 1994 & $0.585(0.019)$ & 0.149 \\
\hline 17 Oct 1994 till 24 Oct. 1995 & $0.784(0.024)$ & 0.384 \\
\hline 24 Oct. 1995 till 1 July 1997 & $0.556(0.029)$ & 0.000 \\
\hline 1 July 1997 till 25 Oct. 1998 & $0.348(0.046)$ & 0.000 \\
\hline \multicolumn{3}{|c|}{$t$-copula degrees of freedom, $\nu$} \\
\hline Period & \multicolumn{2}{|c|}{$\hat{\nu}$ (s.e.) } \\
\hline 27 April 1986 till 30 Oct. 1989 & \multicolumn{2}{|c|}{$5.220(1.075)$} \\
\hline 8 Nov. 1989 till 26 Sept. 1994 & \multicolumn{2}{|c|}{$8.570(2.408)$} \\
\hline 17 Oct. 1994 till 16 Oct. 1995 & \multicolumn{2}{|c|}{$6.215(3.049)$} \\
\hline 24 Oct. 1995 till 25 Oct. 1998 & \multicolumn{2}{|c|}{$+\infty$} \\
\hline
\end{tabular}

Estimated correlation and degrees of freedom between the change-points detected, using the $t$-copula model for the dependence between USD-DEM and USD-JPY. The third column of the upper panel of the table has the coefficient of tail dependence values obtained from the estimated model parameters.

\subsection{Specification tests and comparison with a benchmark model}

After having estimated the number and the location of change-points for the correlation and degrees of freedom in the $t$-model we have to check if the model for the dependence structure is well specified. In order to check this, we have to repeat the procedure of choosing the best copula model applied in Section 3.2 for the full sample, but now for each sub-sample between the change-points. Again, as the change-points estimated for the degrees of freedom are close to change-points estimated for the correlation, we exclude the small periods between those just as in Section 3.3. The best BIC ranked model after fitting the eleven models listed in Table 4 to each sub-sample are reported in Table 8 . The results confirm that the $t$-copula model is the best choice among the proposed models. For the observations after October 1995 the best model is the Gaussian copula. This confirms the infinitely large degrees of freedom estimate for the period after October 1995 reported in Table 7. We recall here that the Gaussian copula is the limit of the $t$-copula when the degrees of freedom of the later go to infinity.

We compare our results obtained by modeling the dependence structure allowing for breaks 
Table 8: Best ranked copula model between the change-points

\begin{tabular}{cc}
\hline \hline \multicolumn{1}{c}{ Period } & Model \\
\hline 27 April 1986 till 29 Dec. 1986 & $t$-model \\
29 Dec. 1986 till 30 Oct. 1989 & $t$-model \\
8 Nov. 1989 till 23 Oct. 1990 & $t$-model \\
23 Oct. 1990 till 26 Sept. 1994 & $t$-model \\
17 Oct. 1994 till 16 Oct. 1995 & $t$-model \\
24 Oct. 1995 till 1 July 1997 & Gaussian-model \\
1 July 1997 till 25 Oct. 1998 & Gaussian-model \\
\hline
\end{tabular}

Results from fitting copula models to the filtered returns of USD-

DEM and USD-JPY between the estimated change-points. For each sub-period between change-points the table has the best ranked model (using the BIC criterion) among the eleven copula models fitted before to the all sample in Section 3.2.

with a benchmark model where the dependence can also be time-varying. A model which allows for this flexibility is the BEKK model from Engle and Kroner (1995). The dependence structure in the BEKK is modeled by a dynamic variance-covariance matrix for which the components change on time. This model can be used with Student- $t$ bivariate innovations which is needed for these data. Although the BEKK model does not allow for time-varying degrees of freedom we can compute the modeled correlation path and compare it with the change-points results. In the case of the BEKK model the degrees of freedom of the multivariate Student- $t$ innovations are constant and the same for all the margins and for the dependence structure. These are two relevant constraints because on one hand the change-point tests revealed the existence of breaks in this parameter and on the other hand, for our data, the degrees of freedom of each margin and of the copula are different as we can see in Tables 2 and 8 respectively. A BEKK model has a large number of parameters which have to be simultaneously estimated. The estimation of this model is difficult especially when the innovations are assumed to be Student$t$ distributed and becomes unwieldy in larger dimensions. The change-points detection is a far more parsimonious approach. In order to use the BEKK we model the conditional mean of each spot rate USD-DEM and USD-JPY returns using the model in Equations (5) and then estimate a bivariate Student- $t$ BEKK model on the residuals. A Gaussian BEKK model was first fitted but did not pass in the goodness-of-fit tests for the residuals. The specification tests for the Student- $t$ BEKK model are reported in Table 9. The residuals pass all the Ljung-Box test for serial correlation and cross-correlation up to the second moment, the test for ARCH effects and the Anderson-Darling goodness-of-fit Student- $t$ test. As expected they fail the normality 
test.

Table 9: Specification tests on the Student- $t$ BEKK filtered returns (p-values)

\begin{tabular}{lccc}
\hline \hline Null hypothesis & USD-DEM & USD-JPY & Multivariate test \\
\hline No serial correlation of 1st moment & 0.8940 & 0.3240 & 0.8555 \\
No serial correl of absol values & 0.7477 & 0.4034 & 0.6010 \\
No serial correlation of 2nd moment & 0.8963 & 0.4838 & 0.8868 \\
No ARCH effects & 0.8724 & 0.4864 & - \\
Normally distributed & 0.0000 & 0.0000 & - \\
Student- $t$ distributed & 0.7260 & 0.1310 & - \\
\hline
\end{tabular}

Here are shown the p-values obtained from Ljung-Box tests for serial correlation and crosscorrelation for the first moments and absolute values of the returns filtered by the Student- $t$ BEKK model. The table also contains the p-values from the ARCH effects LM test, the normality Jarque-Bera and Student-t Anderson-Darling goodness-of-fit tests for the same filtered returns of USD-DEM and USD-JPY.

To visualize the results we display in Figure 1 the time-varying correlation path estimated from the BEKK model, super-imposed with the estimated change-point cross-correlation for the seven periods between the times of change. The BEKK correlation path is extremely jagged. We pose the question how much of this dynamics in the correlation are actually noise. On the other hand, by construction the path for the correlation given by the change-points is constant between those. The change-point analysis seems to detect the main features of the changes in the dynamic correlation curve and to ignore smaller changes. 


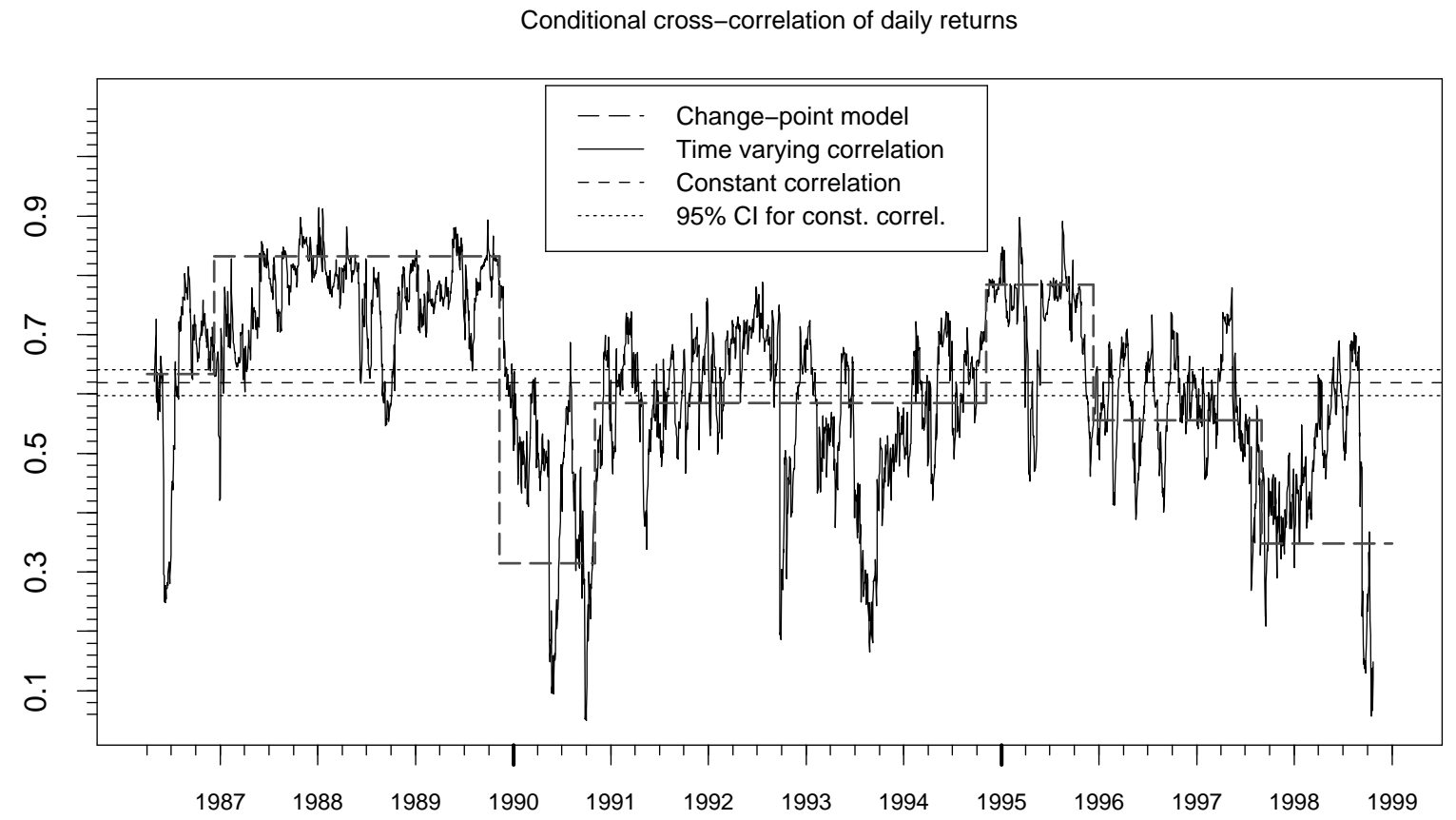

Figure 1: Estimated correlation paths of daily returns on the FX USD-DEM and USD-JPY spot rates. The long-dashed line is the estimated correlation by the change-point tests. This is super-imposed on the estimated correlation using the time-varying Student- $t$ BEKK model from Engle and Kroner (1995). The short-dotted line is the time-invariant correlation estimate after the univariate GARCH filtering. The change-points model reacts quicker to important economic events as the fall of the Berlin wall in November 1989 than the time-varying model and ignores smaller possible changes given by the BEKK model. 


\section{Conclusion}

The aim of the paper is essentially twofold. First of all, we want to contribute to the ongoing discussion between practitioners and academics in order to advance the methodological basis for risk measurement technology. The non-linear copula based model presented in this paper contributes to this goal. Second, through the example of the two-dimensional FX data, we have demonstrated a parsimonious conditional dependence model that takes changes in the dependence structure into account. Evidence for the existence of changes in the conditional correlation between foreign exchange spot rates as been reported in the literature; see for instance Andreou and Ghysels (2003). Economic events, changes in the economic variables or central bank measures are liable of producing changes in the co-movements of prices in financial markets and in particular in foreign exchange rates. We present a methodology which combines the use of change-point tests and the decomposition of the multivariate distribution of asset prices in its marginal distributions and dependence structure. Like this we are able to detect structural changes without having to assume a priori a possible date for its occurrence. Further we can detect changes beyond linear correlation which nevertheless have an important impact in relevant risk measures, in pricing or in asset allocation. That is the case for changes found in the heaviness of the tail of the multivariate distribution. The procedure consists on modeling the univariate marginal dynamics with ARMA-GARCH models and testing for changes in the conditional dependence modeled with parametric copula models. The result is a two stage parsimonious procedure easier to estimate although able to model dynamics beyond what can be captured by benchmark models as the DCC-GARCH or the BEKK from Engle (2002) and Engle and Kroner (1995) respectively. Applying the methodology presented we find changes in the correlation and in the heaviness of the tail of the dependence between Deutsche Mark and Japanese Yen modeled by a $t$-copula. The change points found relate to specific economic events. The most relevant ones are a drop in the correlation and in the heaviness of the tail related with the fall of the Berlin wall in 1989. Another is a strengthening in the correlation around the burst in the Japanese asset price bubble in the fall of 1990 .

\section{Acknowledgements}

We acknowledge useful discussions with Alexander McNeil, and anonymous referees and the journal editor for several detailed comments on an earlier version of this paper. 


\section{References}

Andersen, T. G., Bollerslev, T., and Diebold, F. X. (2005). Parametric and nonparametric volatility measurement. In: Handbook of Financial Econometrics (Ed. L. P. Hansen and Y. Ait-Sahalia), Amsterdam: North-Holland.

Andreou, E. and Ghysels, E. (2003). Tests for breaks in the conditional co-movements of assets returns. Statistica Sinica, 13:1045-1073.

Bai, J. (1997). Estimating multiple breaks one at a time. Econometric Theory, 13:551-563.

Bai, J. and Perron, P. (1998). Estimating and testing linear models with multiple structural changes. Econometrica, 66:47-78.

Bollerslev, T., Chou, R. Y., and Kroner, K. (1992). ARCH modeling in finance. Journal of Econometrics, 52:5-59.

Boyer, B. H., Gibson, M. S., and Loretan, M. (1999). Pitfalls in tests for changes in correlations. Board of Governors of the Federal Reserve System, International Finance Discussion Paper, no. 597 .

Breymann, W., Dias, A., and Embrechts, P. (2003). Dependence structures for multivariate high-frequency data in finance. Quantitative Finance, 3:1-14.

Chen, X. and Fan, Y. (2005). Estimation of copula-based semiparametric time series models. Journal of Econometrics, 130(2):307-335.

Cherubini, U., Luciano, E., and Vecchiato, W. (2004). Copula Methods in Finance. Wiley, Chichester.

Choi, K. and Zivot, E. (2007). Long memory and structural changes in the forward discount: An empirical investigation. Journal of International Money and Finance, 26(3):342-363.

Csörgő, M. and Horváth, L. (1997). Limit Theorems in Change-Point Analysis. Wiley, Chichester.

Daul, S., Giorgi, E. D., Lindskog, F., and McNeil, A. (2003). Using the grouped t-copula. RISK Magazine, pages 73-76.

Demarta, S. and McNeil, A. J. (2005). The t copula and related copulas. International Statistical Review, 73(1):111-129. 
Dias, A. and Embrechts, P. (2002). Change-point analysis for dependence structures in finance and insurance. In: Novos Rumos em Estatística (Ed. C. Carvalho, F. Brilhante and F. Rosado), Sociedade Portuguesa de Estatística, Lisbon, pages 69-86. Also in: Risk Measures for the 21st Century (Ed. G. Szegö), John Wiley and Sons, New York (2004), Chapter 16, pages $321-335$.

Diebold, F. X. and Inoue, A. (2001). Long memory and regime switching. Journal of Econometrics, 105(1):131-159.

Embrechts, P., McNeil, A. J., and Straumann, D. (2002). Correlation and dependence in risk management: Properties and pitfalls. In: Risk Management: Value at Risk and Beyond (Ed. M. Dempster), Cambridge University Press, Cambridge, pages 176-223.

Engle, R. (2002). Dynamic conditional correlation: A simple class of multivariate generalized autoregressive conditional heteroskedasticity models. Journal of Business and Economic Statistics, 20(3):339-350.

Engle, R. and Sheppard, K. (2001). Theoretical and empirical properties of dynamic conditional correlation MVGARCH. Working Paper No. 2001-15, University of California, San Diego.

Engle, R. F. (1982). Autoregressive conditional heteroscedasticity with estimates of the variance of United Kingdom inflation. Econometrica, 50(4):987-1007.

Engle, R. F. and Kroner, K. F. (1995). Multivariate simultaneous generalized ARCH. Econometric Theory, 11(1):122-150.

Fortin, I. and Kuzmics, C. (2002). Tail-dependence in stock return-pairs. International Journal of Intelligent Systems in Accounting, Finance \& Management, 11(2):89-107.

Francis, N. and Owyang, M. T. (2005). Monetary policy in a markov-switching VECM: Implications for the cost of disinflation and the price puzzle. Journal of Business and Economic Statistics, 23(3):305-313.

Genest, C., Ghoudi, K., and Rivest, L.-P. (1995). A semiparametric estimation procedure of dependence parameters in multivariate families of distributions. Biometrika, 82(3):543-552.

Giacomini, E., Härdle, W. K., Ignatieva, E., and Spokoiny, V. (2006). Inhomogeneous dependency modelling with time varying copulae. Weierstrass Institute for Applied Analysis and Stochastics, SFB 649 Discussion Paper 2006-075. 
Gombay, E. and Horváth, L. (1999). Change-points and bootstrap. Environmetrics, 10:725736.

Hamilton, J. D. (1990). Analysis of time series subject to changes in regime. Journal of Econometrics, 45(1-2):39-70.

Hansen, B. E. (2001). The new econometrics of structural change: Dating breaks in U.S. Labor Productivity. Journal of Economic Perspectives, 15:117-28.

Hosking, J. R. M. (1980). The multivariate portmanteau statistic. Journal of the American Statistical Association, 75(371):602-608.

Joe, H. (1997). Multivariate Models and Dependence Concepts. Chapman \& Hall, London.

Longin, F. and Solnik, B. (2001). Extreme correlation of international equity markets. Journal of Finance, LVI(2):649-676.

Loretan, M. and Phillips, P. C. B. (1994). Testing the covariance stationarity of heavy-tailed time series: An overview of the theory with applications to several financial data sets. Journal of Empirical Finance, 1(2):211-228.

McNeil, A. J., Frey, R., and Embrechts, P. (2005). Quantitative Risk Management: Concepts, Techniques and Tools. Princeton University Press, Princeton, New Jersey.

Mikosch, T. and Stărică, C. (2000). Is it really long memory we see in financial returns? In: Extremes and Integrated Risk Management (Ed. P. Embrechts), Risk Books, Waters Group, London, pages 149-168.

Mikosch, T. and Stărică, C. (2004). Non-stationarities in financial time series, the long range dependence and the IGARCH effects. Review of Economics and Statistics, 86(1):378-390.

Nelsen, R. B. (2006). An introduction to copulas. Springer Series in Statistics. Springer, New York, second edition.

Patton, A. J. (2006a). Estimation of multivariate models for time series of possibly different lengths. Journal of Applied Econometrics, 21:147-173.

Patton, A. J. (2006b). Modelling asymmetric exchange rate dependence. International Economic Review, 47(2):527-556. 
Pesaran, M. H., Schuermann, T., and Weiner, S. M. (2004). Modeling regional interdependencies using a global error-correcting macroeconometric model. Journal of Business Economics and Statistics, 22(2):129-162.

Polzehl, J. and Spokoiny, V. (2006). Varying coefficient GARCH versus local constant volatility modeling. Comparison of the predictive power. Weierstrass Institute for Applied Analysis and Stochastics, SFB 649 Discussion Paper 2006-033.

Rockinger, M. and Jondeau, E. (2006). The copula-GARCH model of conditional dependencies: An international stock-market application. Journal of International Money and Finance, $25(5): 827-853$.

Rosenberg, J. V. and Schuermann, T. (2006). A general approach to integrated risk management with skewed, fat-tailed risks. Journal of Financial Economics, 79(3):569-614.

Sklar, A. (1959). Fonctions de répartition à $n$ dimensions et leurs marges. Publications de l'Institut de Statistique de L'Université de Paris, 8:229-231.

Vostrikova, L. J. (1981). Detecting "disorder" in multidimensional random processes. Soviet Mathematics Doklady, 24(1):55-59. 